\title{
De la torre de marfil a la arena pública: El papel de los científicos en la comunicación de la ciencia y la tecnología
}

\section{From the ivory tower to the public arena: the role of scientists in science and technology communication}

\author{
Autora \\ Victoria E. Mendizábal \\ https://orcid.org/0000-0002-3436-748X \\ Facultad de Ciencias de la Comunicación \\ Universidad Nacional de Córdoba \\ Córdoba, Argentina
}

\section{Resumen}

La clásica noción de ciencia neutral y exenta de responsabilidades por las posibles consecuencias problemáticas de los resultados de la investigación científica, poco a poco se ha ido erosionando. En este nuevo escenario, los científicos se están viendo obligados a salir de su tradicional refugio, el laboratorio, para empezar a rendir cuentas a la sociedad de manera sistemática. El presente artículo analiza el contexto en el que estas transformaciones han tenido lugar en la historia más reciente. Asimismo, reflexiona acerca de los motivos por los que las organizaciones científicas comienzan a promover la idea de que la comunicación de los resultados de las investigaciones al gran público, es una tarea que debe ser incorporada al quehacer científico cotidiano. Además, plantea la necesidad de brindar a los científicos herramientas específicas a la hora de comunicar los resultados de sus investigaciones a un público no experto. También propone repensar las actividades de comunicación pública de la ciencia, amplian-

\section{Abstract}

The classical notion of science as being neutral and devoid of responsibilities for the problematic consequences of the results of scientific research has been progressively eroded. In this new context, scientists are being compelled to go outside their traditional refuge, the lab, and render account to society, systematically. The present paper analyzes the context in which these transformations have occurred in the most recent history. Moreover, it reflects about the reasons why scientific organizations have begun to promote the idea that communicating research results to the general public, is a task that must be incorporated to day-to-day scientific endeavor. Furthermore, it raises the need to provide scientists the specific tools to communicate their research results to a non-expert public. In addition, it invites to reconsider public communication of science activities beyond scientific journalism practice. Finally, it suggests that the challenge of communicating research results to wider audiences im- 
do estas prácticas más allá del periodismo científico. Finalmente, sugiere que el desafío de comunicar los resultados de sus investigaciones a un público amplio, supone encontrar estrategias que permitan a los científicos romper la estructura tradicional de comunicación con sus pares, mayoritariamente argumentativa, y llevarla a un formato narrativo.

Palabras clave: Comunicación pública de la ciencia, científicos, responsabilidad social, habilidades de comunicación, investigación, narrativas plies finding strategies that allow scientists to break their traditional forms of peer-to-peer communication, mainly argumentative, and transform them into narratives.

Keywords: Public communication of science, scientists, social responsibility, communication skills, research, narratives

Hay quienes sostienen que la denominada torre de marfil en la que los científicos se han refugiado durante siglos, se está desmoronando. Para autores como Miguel Alcíbar (2016), la metáfora acuñada por el escritor y crítico literario francés Charles Augustin Sainte-Beuve en el siglo XIX, ya no resultaría operativa a la hora de resguardar a los científicos de los problemas mundanos.

Como muchos autores coinciden en señalar, la clara demarcación entre el ámbito de la ciencia -como conocimiento y constatación objetiva de la realidad- y el ámbito de los valores, las normas, las ideologías y los intereses, se ha ido deteriorando en las últimas décadas. Como veremos a continuación, la clásica noción de ciencia, neutral y exenta de responsabilidades por las posibles consecuencias problemáticas de los resultados de la investigación científica, poco a poco se ha ido erosionando. En este contexto, los científicos se están viendo obligados a salir de su clásico refugio, el laboratorio, para empezar a rendir cuentas de cara a la sociedad de un modo sistemático.

En palabras de Shirley Williams, parece que «la fiesta ha terminado para los científicos». En un artículo que publicó en febrero de 1971 en la revista The Times, esta política británica de renombre pronosticaba el fin de las clásicas políticas científicas de laissez-faire en las que la financiación incondicional de la ciencia se basaba en el optimismo sobre sus potencialidades como proveedora de bienestar y progreso. Como consecuencia de la acumulación de catástrofes relacionadas con la aplicación de la ciencia y la tecnología y el desarrollo de movimientos sociales críticos, se produce una crisis de confianza tal que las políticas públicas sobre ciencia y tecnología se abren a la intervención activa de los gobiernos y el atento escrutinio de otros agentes sociales. Pero, ¿cómo es que llegamos hasta aquí?

\section{Ciencia y tecnología bajo la lupa}

La noción tradicional que aún hoy predomina en muchos sectores y que considera a la ciencia básica motivada por valores exclusivamente epistémicos, queda muy bien reflejada en los dichos del Galileo construido por el dramaturgo alemán Bertolt Brecht en su obra Vida de Galileo (1938-1939). «Yo he escrito un libro sobre Mecánica del Universo y eso es todo. Lo que se haga o no se haga con eso, no me interesa», dice Galileo y reafirma la idea de que es posi- 
ble interpretar a cualquier innovación o procedimiento tecnológico como una mera aplicación de conocimientos científicos, cuyo desarrollo tiene en la eficacia y en la eficiencia sus únicos valores.

Como si se tratara de un efecto automático, la acumulación de conocimiento científico mediante un proceso que se inicia en la investigación básica, se traduce en el desarrollo de productos tecnológicos de los cuales la sociedad es la beneficiaria y el único escenario de posibles disputas valorativas. De acuerdo con esta concepción, existiría una relación lineal entre la ciencia, la tecnología y la sociedad (CTS), los elementos que conformarían los tres eslabones perfectamente delimitados de una cadena bien ordenada. Una concepción que dio sustento al llamado modelo unidireccional de desarrollo y que predominó desde la Segunda Guerra Mundial, sirviendo de base a las políticas públicas sobre ciencia y tecnología (López Cerezo, 1999).

Figura 1. Esquema del modelo unidireccional

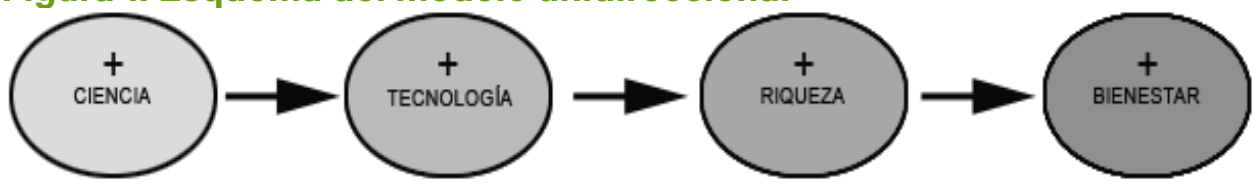

Así, el nuevo modelo de organización de la ciencia representado por el Proyecto Manhattan (un proyecto de investigación científica desarrollado entre 1939 al 1946 por Estados Unidos con el apoyo del Reino Unido y de Canadá para desarrollar la primera bomba atómica) fortaleció la idea de que con mucho dinero, objetivos claros, científicos brillantes y liderazgo adecuado podía conseguirse casi cualquier objetivo que un gobierno considerara importante. En la medida en que es concebida como un recurso estratégico, la ciencia comienza a ser objeto de un conjunto de medidas orientadas a desarrollar su potencial.

A partir de la producción de penicilina, la invención del radar o la fabricación de la bomba atómica, el acervo de ciencia básica disponible se transforma para los Estados desarrollados en la condición necesaria para el logro de objetivos nacionales. Ejemplo de ello es el informe Ciencia, la frontera sin fin (1945), un encargo del presidente Roosevelt elaborado por Vannevar Bush junto con otros científicos en el que se propuso cómo aprovechar la experiencia acumulada durante la guerra para servir en tiempos de paz.

Este informe sistematiza el llamado 'modelo lineal de política científica y tecnológica.' Este modelo considera que la posibilidad de innovación tecnológica y el desarrollo de productos con valor económico, estratégico o social están directamente relacionados con la capacidad de investigación básica. De alguna manera, este modelo reforzó una especie de optimismo incondicional hacia el progreso científico como sinónimo de bienestar social (sobre todo en Estados Unidos). Sin embargo, también despertó una actitud crecientemente crítica y cautelosa hacia la ciencia y la tecnología que comenzó a extenderse sobre todo a partir de los años sesenta. Está claro que la organización estatal y militar de la investigación científica durante la guerra condujo a resultados decisivos, de los cuales el más espectacular fue la construcción de bombas atómicas en el Proyecto Manhattan. Pero, al mismo tiempo, los bombardeos de Hiroshima y Nagasaki también despertaron las conciencias de algunos de los científicos e intelectuales más influyentes de la época. 
Tal es el caso del filósofo y matemático británico Bertrand Russell, quien ya en 1945 manifestó su cautela en cuanto a las consecuencias del uso de la energía nuclear. Asimismo, Józef Rotblat, el único científico que abandonó el Proyecto Manhattan por cuestiones morales, afirmó que se hallaba preocupado por el futuro de la humanidad, después de saber del bombardeo de Hiroshima y viendo la inminente escalada armamentística nuclear. Más adelante, tanto Russell como Rotblat trabajaron juntos y en colaboración con Albert Einstein y otros científicos para evitar la proliferación de armas nucleares y, finalmente, constituir el Manifiesto Russell-Einstein en $1955^{1}$

Años más tarde, también fundaron las Conferencias Pugwash en 1957, una serie de conferencias internacionales sobre ciencia y asuntos mundiales convocadas con el fin de discutir asuntos tales como el desarme y la responsabilidad social del científico en temas como el crecimiento demográfico, el deterioro medioambiental y el desarrollo económico². Inclusive, es interesante destacar que el propio Brecht elabora una nueva versión de Vida de Galileo a partir de observar el impacto de las bombas. Y pone en boca de Galileo una seria advertencia sobre los usos de la investigación científica:

«Si los científicos, intimidados por los poderosos egoístas, se contentan con acumular Ciencia por la Ciencia misma, se la mutilará, y vuestras nuevas máquinas significarán solo nuevos sufrimientos. Quizás descubráis con el tiempo todo lo que haya que descubrir, pero vuestro progreso será solo un alejamiento progresivo de la Humanidad. El abismo entre vosotros y ella puede ser un día tan grande que vuestros gritos de júbilo por alguna nueva conquista sean respondidos por un griterío de espanto universal...». Vida de Galileo, Bertolt Brecht (1945-1947)

A partir de la década del 60, esta creciente preocupación por parte de algunas élites intelectuales también se vio reflejada en diferentes movimientos sociales que empezaron a considerar a la ciencia y la tecnología como objetos de escrutinio público y debate político.

Alimentados por catástrofes relacionadas con la tecnología, tales como accidentes nucleares, envenenamientos farmacéuticos o derramamientos de petróleo, se fueron desarrollando activos movimientos sociales críticos con el industrialismo y el estado tecnocrático. Asimismo, el desarrollo del movimiento ecologista y las protestas públicas contra el uso civil y militar de la energía nuclear fueron elementos importantes de esa reacción desde finales de los años sesenta y comienzos de los setenta.

Es precisamente en este contexto y como consecuencia de este incremento de la participación pública que, desde entonces, comienza a revisarse el citado modelo unidireccional de desarroIlo. De aquí surgen, en los años setenta, instrumentos como la evaluación de tecnologías y de impacto ambiental, e instituciones calificadoras y reguladoras adscritas a distintos poderes en diferentes países (González García y cols., 1996).

Coincidiendo con estas respuestas intelectuales, sociales y políticas, también se produjeron cambios afines en algunas disciplinas académicas tradicionales como la sociología, la historia y la filosofía de la ciencia y de la tecnología. Unos cambios sobre los que la obra de autores como Thomas Kuhn tuvo una enorme influencia. De manera más o menos independiente, sociólogos, historiadores y filósofos se alejaron de los estudios internalistas y comenzaron 
a hacer interpretaciones más contextualistas, con un factor común: la crítica a las nociones tradicionales de 'objetividad' dentro del conocimiento científico y tecnológico.

No se trataba de negar por completo la 'realidad' de la naturaleza o de los artefactos, se pretendía insistir sobre el hecho de que nuestra comprensión de la naturaleza y la creación de tecnología eran procesos socialmente mediados (Cutcliffe, 2003).

En resumen, desde los tiempos de Galileo en los que el científico era un artesano que fabricaba sus propios instrumentos para realizar experimentos y obtener evidencias que le permitieran describir la realidad, llegamos a una suerte de científico emprendedor que debe situarse en una lógica de competencia por los recursos y los espacios. La posibilidad de acceder a estos recursos marca la diferencia a la hora de realizar experimentos que de otro modo no serían posibles. En este punto, la fuerte inversión por parte de los Estados y los intereses de la industria comienzan a borrar la línea entre investigación privada y pública.

Así, esta nueva manera de encarar la investigación científica entra en conflicto con la visión tradicional de la ciencia que presupone el carácter autónomo del conocimiento científico. Un enfoque clásico que descansa en el supuesto de que el conocimiento científico es producido por individuos inmersos en subculturas incomprensibles para el público general, mediante la observación y la manipulación desinteresada de la naturaleza y la posterior evaluación racional de sus descubrimientos. Por eso, no resulta extraño que si bien, como decíamos al principio, la ciencia sigue gozando del prestigio social, las actitudes hacia ella oscilan hoy, en palabras del epistemólogo Wartofsky, «entre la veneración burda y la burda sospecha» (1976).

\section{Tensiones y razones entre ciencia y comunicación}

Escribir y publicar son dos de las principales tareas regulares del científico. De hecho, solo cuando este hace públicas sus investigaciones, es que puede terminar de convertirlas en 'ciencia' a través de la crítica de sus pares y de permitir la replicabilidad de sus experimentos.

Su carácter público ha sido un rasgo distintivo de la ciencia moderna desde sus orígenes. En efecto, formalizada por Merton (1973) como una de las normas básicas de la ciencia académica, el comunitarismo se refiere a la necesidad de hacer públicos los resultados de las investigaciones para favorecer la comunicación entre los miembros de la comunidad científica.

Sin embargo, la relación entre los científicos y el público -o más específicamente, entre los científicos y los medios de comunicación- siempre se ha caracterizado con términos como "distancia", "brecha", "barrera", "cerco", "agua y aceite" y en el menos conflictivo de los escenarios como una "tensión creativa" (Peters, 2013).

En un ya clásico artículo "Una relación difícil: las tensiones entre la medicina y los medios de comunicación" (1996), la socióloga Dorothy Nelkin señala que muchos de los conflictos entre científicos y periodistas, están vinculados a sus distintos modos de explicar las noticias científicas y sus diferentes estilos de divulgación. Por encima de todo, Nelkin lo atribuye a la falta de acuerdo sobre el papel de los medios de comunicación en la difusión del conocimiento especializado. Así, en muchos científicos subyace la idea de que los medios de comunicación 
deberían funcionar como una simple tubería o conducto que transmite la ciencia al público haciendo que sea fácilmente comprensible.

En contraste, la vocación de analista crítico de la realidad del periodista hace que los argumentos científico-técnicos se diluyan cuando incluye discusiones acerca de sus implicaciones sociales, o al cuestionar los límites éticos de los descubrimientos. En esta línea, Reed (2001), sostiene que los periodistas se hallan más comprometidos con la crítica, el entretenimiento y la información, que con la comunicación académica y la alfabetización pública promovida por los científicos. Esto choca con la creencia de muchos científicos de que el propósito del periodismo científico es transmitir una imagen positiva de la ciencia. Una ciencia a la que consideran alejada de valores humanos y que está únicamente sustentada en valores epistémicos como la objetividad o la verdad.

Está claro que los periodistas científicos no han tenido más remedio que aprender a lidiar con la jerga especializada, la complejidad de las metodologías experimentales, la rigurosidad de las revistas científicas y hasta con los egos de algunos investigadores. Es parte de su trabajo y lo saben. En contraste, para la mayoría de los científicos, la comunicación con la prensa o el público es vista con frecuencia como una actividad que interfiere con el trabajo real de investigación y que no resulta en una remuneración ni en un reconocimiento por parte del sistema científico. Esto se suma a la reticencia natural de los científicos a lidiar con un público al que consideran impredecible y al temor de perder credibilidad ante unos colegas que podrían pensar que lo hacen buscando publicidad. Asimismo, a numerosos científicos les preocupa además la interpretación incorrecta de los resultados de sus investigaciones y la simplificación excesiva de los temas de investigación.

Sin embargo, datos más recientes muestran que, al menos en los países líderes en investigación y desarrollo, las interacciones entre los científicos y los medios de comunicación serían más frecuentes y menos conflictivas de lo que se pensaba. Según revela una encuesta que compara los resultados obtenidos en Estados Unidos, Japón, Reino Unido, Alemania y Francia, cerca del $70 \%$ de los científicos encuestados afirma que ha tenido algún tipo de contacto con los medios en los últimos tres años (Peters y cols., 2008). Asimismo, los investigadores con mayor producción científica y roles de liderazgo perciben que la interacción con los medios tiene un saldo positivo. Si bien se sienten inseguros y que no controlan la situación dada 'la imprevisibilidad de los periodistas', admiten que a través de su interacción con la prensa buscan generar en el público una actitud más positiva hacia la investigación. Más aún, a pesar de que un $42 \%$ de los encuestados temen reacciones negativas por parte de sus pares, una proporción similar (39\%) afirma que sus relaciones con la prensa contribuyeron a una mejor reputación personal entre sus colegas.

En esta línea, un reciente estudio en el que se entrevista a cerca de 3.748 científicos estadounidenses elegidos al azar entre los miembros de la American Association for the Advancement of Science (AAAS), revela que la comunidad científica se enfrenta a cambios que incluyen, entre otras cosas, salir de su zona de confort y ser más protagonista en la sociedad. Fundamentalmente, a fin de hacer sus proyectos más visibles en momentos en los que hay serias dificultades para conseguir financiación. Según la encuesta, el $87 \%$ de los científicos opina que debe ser más activo en los debates de políticas públicas. El 43\% piensa que un mayor protagonismo 
en los medios de comunicación y en las noticias puede ser positivo para sus carreras, frente al $37 \%$ que opinaba esto en una encuesta de 2009 (Pew, 2015)

En este contexto, promover una interacción efectiva entre científicos y el público general no podría ser más oportuno y pertinente. El sector de investigación está permanentemente produciendo hallazgos científicos que no sólo contribuyen al avance de nuestro conocimiento acerca del mundo, sino que además tienen el potencial de transformar las sociedades y la vida de las personas, planteando -en muchos casos- conflictos y dilemas que generan intensos debates en la esfera pública.

Estos nuevos escenarios, hoy posibles o en construcción, rozan cada vez con mayor fuerza los límites éticos y han propiciado la aparición de nuevos actores sociales que reclaman participación en las decisiones -tanto individuales como colectivas- vinculadas al desarrollo tecno-científico. Pacientes que reclaman autonomía, consumidores que luchan por sus derechos, ciudadanos que demandan mayor participación en la formulación de políticas públicas como el desarrollo de la energía nuclear, la megaminería o la expansión de las fronteras agrícolas, habitantes del planeta que quieren heredar a sus hijos los mismos recursos que recibieron.

Por otro lado, el progreso científico resulta central en muchos de los principales retos de nuestras sociedades: hacer frente al cambio climático, responder a la creciente demanda mundial de alimentos, enfrentar los desafíos de los nuevos usos y aplicaciones de la biomedicina, fomentar el desarrollo de estilos de vida sustentables. Todo ello en un contexto en el que la incidencia de diversos determinantes sociales (y políticos) resulta crítica a la hora de abordar diversas problemáticas.

Es claro que compartiendo sus conocimientos acerca del mundo natural y social, los científicos pueden ayudar al público en la toma de decisiones, tanto individuales como colectivas, acerca de problemáticas complejas. De modo similar, el público puede enriquecer la práctica científica ofreciendo perspectivas novedosas, contribuyendo en la sintonía fina de la investigación actual o estimulando nuevas líneas de investigación.

Así lo expresa, por ejemplo, un informe del grupo de trabajo creado por la Comisión Europea para establecer el significado de la participación y el diálogo entre los diferentes actores sociales y la comunidad científica: «Los procesos de innovación exitosos requerirán no solamente la cooperación entre academia y empresa, sino también la activa participación de la sociedad. Esto no solo moldeará la innovación actual sino que también servirá para identificar necesidades futuras» (EURAB, 2007).

Por todo lo expuesto, y más allá de ciertas resistencias, poco a poco, los científicos empiezan a comprender que deben comunicar los resultados de sus investigaciones a diversos públicos por fuera de su campo de influencia más directo. Esto es así, por motivos que podríamos agrupar en tres grandes colecciones de argumentos: éticos, de sostenibilidad y de licencia social. 


\section{Figura 2. Razones para comunicar}

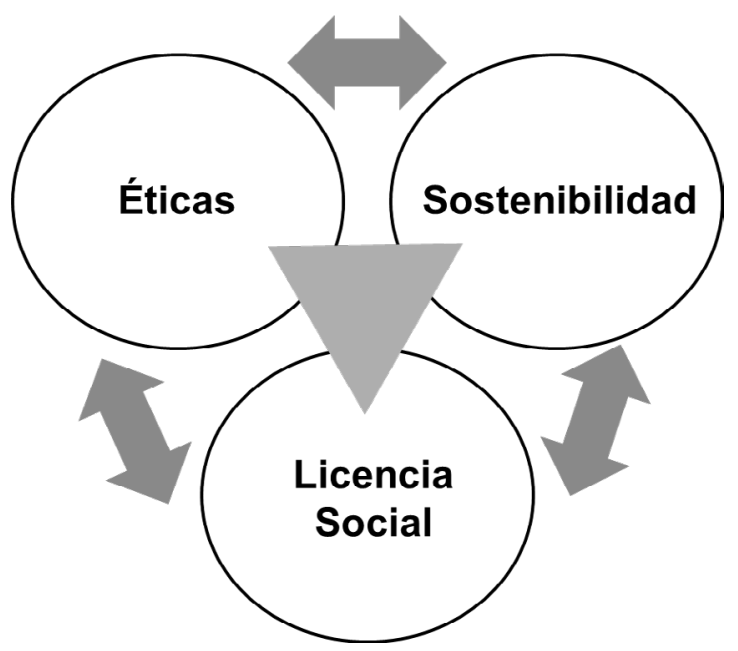

A lo largo de la historia de la ciencia, el deseo de compartir su conocimiento con el gran público siempre ha sido una buena razón para los científicos. Por aquello de que "es bueno en sí mismo tener cultura científica", porque "es necesario que la población esté informada acerca de los últimos descubrimientos de la ciencia", porque "es preciso democratizar el conocimiento", porque "hay que devolverle al pueblo la inversión en ciencia y tecnología que realiza a través de sus impuestos" y un sinnúmero de respuestas más o menos conocidas, más o menos utilizadas como clásicos caballitos de batalla.

Más recientemente, este conjunto de razones que podríamos denominar éticas, han adquirido otra dimensión, vinculada al concepto de responsabilidad social. En particular, y en gran parte como consecuencia del impacto del Proyecto Manhattan, a partir de la segunda mitad del siglo XX se ha producido un cambio sustancial en las actitudes de algunos científicos frente a su responsabilidad, a la hora de pensar (y comunicar) los usos y aplicaciones de sus descubrimientos. Así, personajes como Albert Eistein y su contribución en la creación de experiencias como las ya mencionadas Conferencias Pugwash o la controvertida influencia de Rachel Carson en la historia del ambientalismo, sin duda han marcado un camino en lo que hoy se conoce como Responsible Reserach and Innovation ${ }^{3 .}$

Por otro lado, en las últimas décadas se han profundizado otro segundo conjunto de razones vinculadas a la sostenibilidad del sistema científico y de las propias líneas de investigación en un escenario cada vez más competitivo por los recursos y en el que resulta cada vez más difícil formar discípulos que quieran continuar el propio trabajo. A una escala más institucional y de política pública, también resulta cada vez más difícil despertar vocaciones científicas capaces de sostener las bases del avance científico y promover el desarrollo de nuevos campos del conocimiento (estratégicos) que han emergido en los últimos años y que necesitan de recursos humanos formados. La consigna parece clara. Tanto a nivel individual como a nivel institucional resulta evidente que para que un determinado campo del conocimiento se desarrolle y se sostenga en el tiempo, la comunicación con sus públicos resulta clave. 
Pensemos en el caso de la NASA, por ejemplo, tal vez la entidad con el sistema de divulgación de la ciencia más importante del mundo. Es indudable que la investigación espacial en EEUU no se sostuvo en el tiempo por mera coincidencia. En palabras de Tomás de Miguel, Director de la RedIRIS:

"Cuando se creó, uno de sus objetivos era poner un hombre en la luna, y el país estaba entusiasmado porque era un proyecto nacional. Una vez que se consiguió, el proyecto continuaba pero la NASA se dio cuenta de que si no eran capaces de transmitir a la sociedad la necesidad de continuar con sus investigaciones el espacio, la institución podía desaparecer por falta de presupuesto" El Mundo, 28/11/2008

Por ello, se creó toda una división con el objetivo de divulgar la ciencia espacial entre los más jóvenes. El resultado: hoy la NASA es una de las instituciones científicas de mayor visibilidad en el mundo y la ciencia espacial, una de las ramas de la ciencia con mayor aceptación social entre jóvenes y adultos..

Finalmente, existe un tercer conjunto de razones vinculadas a lo que algunos autores han denominado licencia social. De un tiempo a esta parte, la ciencia se ha visto implicada en una serie de transformaciones que han excedido los límites del laboratorio, instalándose en la opinión pública como argumento fundamental en una serie de debates sociales, económicos, éticos e incluso políticos.

¿Qué dice la ciencia con respecto a la responsabilidad humana en el cambio climático? ¿Existen realmente nuevas propiedades o funcionalidades de los alimentos o no son más que una estrategia de venta de las grandes multinacionales? ¿Es ético el uso de células madre provenientes de embriones humanos para la investigación? ¿Cuál es el impacto ambiental y social a largo plazo del uso de agroquímicos en la controvertida área de la biotecnología aplicada a la agricultura?

En este contexto, comunicarse con el gran público parece indispensable para aumentar la credibilidad de ciertos sectores científicos, legitimar las actividades de investigación en algunos campos del conocimiento que generan fuertes debates, promover una imagen positiva frente a la opinión pública o lograr ser fuente de los medios de comunicación para así tener más chances de influir, de alguna manera, en las informaciones que le llegan a la sociedad.

\section{La necesidad de profesionalización}

Desde aquel primer gran divulgador de la historia encarnado en Galileo Galilei, hasta personajes más recientes como Carl Sagan o Jacques Cousteau, cuando pensamos en los científicos que promueven el diálogo con el gran público, seguramente advertimos en ellos su profunda vocación por comunicar aquello sobre lo que investigan. Sin duda, la influencia de estos personajes icónicos de la infancia de cualquiera que creció entre finales de los 70 y principios de los 80 , ha sido -en muchos casos- decisiva a la hora de elegir una vocación científica.

¿Quién no se planteó seriamente alguna vez estudiar Biología Marina, cautivado por las extraordinarias dotes de Cousteau para enseñarnos todo acerca del mar, o no soñó con dedicarse a la Astronomía o la Astrofísica mientras seguía, capítulo a capítulo, las aventuras que nos presentaba Sagan en su mítico Cosmos? 
Ya en una relación espacio-temporal más cercana, tenemos versiones actuales y locales de científicos-divulgadores como Adrián Paenza, el hombre que se permitió sacar a las matemáticas de su clásico lugar de cuco de la escuela, o a un Diego Golombek que con su personaje de Proyecto $\mathrm{G}$ nos invita a pensar los experimentos como actividades que pueden estar al alcance de cualquiera.

Resulta evidente que nos referimos a personas con habilidades innatas -y también aprendidas luego de un intenso ejercicio cotidiano- para hacer llegar el conocimiento científico al público de a pie, mediante un lenguaje claro, cercano e incluso en clave de entretenimiento. Ahora bien, ¿se trata de personajes tan únicos e irrepetibles que hay que clonar para que puedan dar abasto con sus innumerables proyectos divulgativos y estar aquí y allá al mismo tiempo, o uno podría pensar que es posible lograr que cualquier científico adecuadamente entrenado podría obtener resultados similares?

En uno de los primeros y más influyentes reportes de la Royal Society, The public understanding of science (1985), se instaba a movilizar a la comunidad científica a salir de los laboratorios y hablar con los medios de comunicación o directamente a un público más amplio acerca de sus investigaciones y promover el entusiasmo por la investigación científica en la sociedad. Precisamente, un monográfico de la revista Public Understanding of Science (PUS, 2011), se propuso recientemente analizar hasta qué punto esta movilización de los científicos hacia la sociedad ha evolucionado en las últimas décadas, desde la aparición del mencionado informe. En este número especial, diversos autores se preguntan hasta qué punto los científicos están involucrados en actividades de comunicación social de la ciencia, en qué ocasiones, con qué intensidad, en qué momentos de sus carreras, en qué proporción, con qué objetivos.

En este marco, no resulta sorprendente que desde hace unos años, la interacción efectiva entre científicos y el público general se esté promoviendo desde algunas instituciones emblemáticas como la Royal Society, la American Association for the Advancement of Science (AAAS) o desde algunos programas de la UE.

En el caso de Argentina, en los últimos años ha desarrollado una política activa en cuanto a las actividades de comunicación pública de la ciencia, especialmente desde la creación del Ministerio de Ciencia, Tecnología e Innovación Productiva en 2008. Iniciativas como la feria Tecnópolis, el canal de ciencia TecTV o el Centro Cultural de la Ciencia, son claros ejemplos de la importancia que se le ha otorgado a la generación de espacios de interacción entre la ciencia y la sociedad. Estas políticas han incluso trascendido los cambios en la administración del Estado, manteniéndose en la nueva gestión de gobierno.

Por otro lado, y en consonancia con este impulso a la comunicación pública de la ciencia, las nuevas generaciones de científicos se encuentran más interesadas y ávidas de participar en actividades de divulgación y de interacción con el gran público. Iniciativas como el grupo de stand up científico Poper o como el proyecto editorial El gato y la caja son un claro ejemplo de este nuevo espíritu divulgador entre los más jóvenes.

Sin embargo, en el ámbito latinoamericano en general y en Argentina en particular, las tareas de comunicación y divulgación aún son vistas como actividades secundarias, de carácter 
vocacional y, en general, no son apoyadas desde las instituciones científicas. Menos aún existen programas de formación en habilidades de comunicación dirigidos a científicos.

Además de la Diplomatura desarrollada por el IDEC-Universidad Pompeu Fabra (De Semir, 2009) en los últimos años se han creado en Argentina, una serie de Especializaciones en Comunicación Pública de la Ciencia, Divulgación y Periodismo Científico en el ámbito de diversas universidades públicas (la Universidad Nacional de Córdoba, la Universidad Nacional de Río Negro y más recientemente la Universidad de Buenos Aires).

Sin embargo, la oferta de estas especializaciones supone una dedicación intensa y extendida en el tiempo que apunta a brindar herramientas a profesionales con distintos bagajes académicos: periodistas y comunicadores sociales, relacionistas públicos, profesionales de la salud, investigadores de diversas áreas de las ciencias experimentales y sociales, entre otros. En este tipo de experiencias educativas, los científicos que trabajan en instituciones de ciencia representan un porcentaje relativamente bajo (cercano a un $20 \%$ ) y, en general, buscan como objetivo reorientar sus carreras o explorar nuevas posibilidades de desarrollo profesional (Mendizábal, 2013).

Por otro lado, este tipo de especializaciones tiene, en general, una fuerte orientación hacia el periodismo en los medios de comunicación, dejando de lado otros aspectos y formatos -a los que nos referiremos más adelante- que son fundamentales de cara a entablar un diálogo con la sociedad. Asimismo, la oferta de formación existente se centra en la comunicación de la ciencia en términos generales y no se orienta a la necesidad de los científicos de comunicar su propia investigación.

A diferencia de lo que ocurre en otras regiones, en las que ya se han puesto en marcha diversas iniciativas que buscan específicamente ayudar a los científicos a comunicar los resultados de sus investigaciones, las tareas de comunicación de cara al público en nuestras latitudes son aún una asignatura pendiente. Experiencias como la del Alan Alda Center for Communicating Science, los workshops para científicos promovidos por la AAAS o incluso los cursos y seminarios organizados desde la Fundación Esteve en España, ni siquiera son vistas como ejemplos a seguir desde las instituciones y organismos de ciencia y tecnología local.

Si bien en los últimos 10 o 15 años poco a poco se va instalando la idea de que la comunicación es un área relevante para el desarrollo científico-tecnológico, aún se confía en el talento y la vocación de unos pocos investigadores que empiezan a meter sus narices en el mundo de la comunicación pública de la ciencia, básicamente, movidos por una inquietud personal. Si bien existen algunas iniciativas que promueven la participación de científicos en actividades de divulgación, tales como Nanotecnólogos por un día, estas no se fundamentan en la necesidad de profesionalizar la comunicación de la investigación científica como una parte esencial de la actividad de investigación, sino en la necesidad de llegar a públicos como los estudiantes secundarios con el fin de promover vocaciones científicas.

En otra línea, desde muchos sectores, aún se sigue viendo a los periodistas especializados como los más idóneos para comunicar la ciencia al gran público. De hecho en los últimos años han proliferado diversas iniciativas de agencias de noticias científicas vinculadas a cen- 
tros de investigación y universidades públicas, lideradas por periodistas y comunicadores, que no se han acompañado de una capacitación de sus científicos en habilidades de comunicación. En muchas ocasiones, la necesidad de disponer de fuentes científicas entrenadas para enfrentar a los medios y los requerimientos de la sociedad sólo se hace evidente en situaciones de emergencia tales como crisis sanitarias, ambientales o la aparición de fenómenos climáticos, entre otros ejemplos.

En resumen, ni en los diversos niveles de la administración pública ni en las organizaciones científicas existe una percepción clara acerca de la necesidad de formar a los científicos en habilidades de comunicación y, por lo tanto, no se destinan recursos para ello.

Por otro lado, si bien en los últimos años organismos de ciencia como el CONICET han incorporado la participación en actividades de divulgación en las plantillas de evaluación de sus investigadores, no existen incentivos claros y concretos hacia aquellos que las realizan. Por otra parte, la gran mayoría de los investigadores percibe que no dispone de las herramientas adecuadas ni los canales para realizar estas tareas.

En este sentido, resulta de interés comentar los resultados encontrados en una encuesta diseñada para explorar las principales dificultades que afrontan los científicos a la hora de comunicar sus resultados a la sociedad (Mendizábal, 2016). Uno de los datos más significativos es que, independientemente del estadio de la carrera científica (becario doctoral, investigador posdoctoral, investigador de planta o líder de grupo) la capacidad de "sintetizar el trabajo de muchos años en pocas líneas" y de encontrar un "lenguaje adecuado para el público objetivo" se encontraron entre las principales preocupaciones de los encuestados.

\section{La continuidad del relato: del cuaderno de laboratorio a la charla de divulgación}

Si bien existe la creencia de que para comunicar una ciencia accesible a todo público hacen falta el talento y la vocación propios de los grandes divulgadores, la comunicación está en el ADN del quehacer científico. Contar las actividades que los científicos realizan en sus laboratorios en un cuaderno de resultados, un seminario interno, un abstract en un congreso o un paper no es tan distinto de contárselo al gran público. Existe una continuidad en esa "narración" aunque cambien la audiencia, el registro y el lenguaje.

Autores como Hilgartner (1990) sostienen que existe un continuum de géneros comunicativos que van desde las discusiones técnicas de laboratorio, en uno de los extremos, pasando por presentaciones orales en congresos científicos y literatura científica publicada, a conferencias y publicaciones dirigidas a un público más amplio por fuera del grupo de pares en el otro extremo, sin que exista una línea divisoria clara entre lo que es "ciencia" y "popularización" de la ciencia. 


\section{Figura 3. Continuum de géneros de comunicación científica. Adaptado de Hilgartner (1990)}

\author{
Dentro de la \\ comunidad
}

Presentación de

poster en congreso

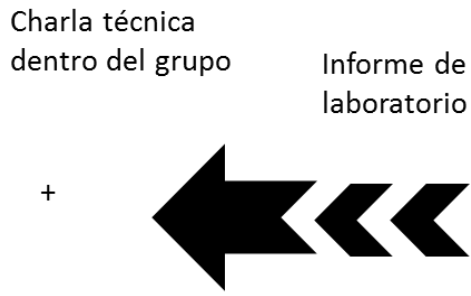

Seminario interno de laboratorio

\section{Comunicación oral} en congreso
Artículo en

revista científica

\author{
Fuera de la \\ comunidad
}

Policy

report

Revisión de la

literatura

científica

\begin{abstract}
Editorial
en revista

científica
\end{abstract}

Libro de

divulgación

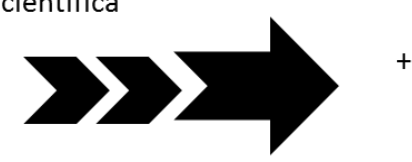

Pedido de

financiamiento texto
Noticia científica
Medios

masivos

Desde esta perspectiva, comunicar es una parte fundamental de la labor de un científico. Cualquier investigador sabe muy bien que preparar una presentación para un seminario interno, un poster para un congreso o escribir un paper, son pasos indispensables para que aquello que ha hecho en el laboratorio, se convierta en "Ciencia".

La ciencia es una práctica tan particular que, si nos hacemos las preguntas correctas, es capaz de darnos respuestas concretas, "certeras" y reproducibles en cualquier laboratorio que se precie, siempre y cuando se sigan los pasos adecuados. De hecho, la reproducibilidad de los resultados de la investigación es una característica distintiva de cualquier resultado con pretensiones de científico. Un resultado que sólo es reproducible si se explica, paso a paso, cómo se ha arribado a él.

Hacer un experimento no se limita a preparar soluciones, correr un gel, tratar células y animales con diversas drogas o realizar medidas con aparatos de alta tecnología. Todo científico está obligado a elaborar un informe escrito de las actividades que desarrolla cada día en su laboratorio y de los resultados obtenidos.

Llevar un cuaderno de laboratorio personal es una práctica hoy bien establecida y sujeta a estrictos controles que implica registrar todo el trabajo experimental realizado y debe contener toda la información necesaria para que otra persona sea capaz de reproducir el experimento, exactamente de la misma forma en la que lo hizo su autor original. Este registro se ha constituido en una prueba esencial, tanto en el mundo de las patentes como frente a la investigación de un posible fraude científico. 
Pero del adecuado registro en el cuaderno de laboratorio a la elaboración del paper, lo cierto es que, en general, no existen instancias de capacitación formal diseñadas para desarrollar habilidades específicas para estas tareas durante la carrera. Es algo que se aprende sobre la marcha y, en muchos casos, a los tumbos. Por ejemplo, es muy probable que la primera vez que un aprendiz de científico se enfrenta a escribir un abstract para un congreso se pase toda una jornada laboral encontrando las 300 palabras adecuadas para que el director de su tesis se lo apruebe. En cambio, un científico entrenado podría escribirlo en 10 minutos, luego de años y años de asistencia a congresos.

Aún dentro de la clásica (y rígida) estructura del discurso científico, el manejo adecuado de recursos retóricos puede resultar crucial a la hora de convencer a editores y revisores de un journal acerca de la imperiosa necesidad de publicar los resultados de una investigación que han costado sangre, sudor y lágrimas. En el universo académico del publish or perish ${ }^{4}$ el desarrollo de las habilidades necesarias para la escritura de artículos científicos puede resultar tan crucial que incluso se han desarrollado capacitaciones específicas para entrenarse, como los que brinda la Fundación Esteve en España desde hace varios años.

El proceso de escritura de un paper es fundante en la formación de un becario doctoral. Más adelante, llegan los informes de avance y los pedidos de financiamiento. Allí se trata de convencer a quienes van a otorgarles el dinero para seguir adelante con sus investigaciones, que les renueven la beca o que les asignen recursos para continuar sus proyectos.

Si tienen suerte, algún día también enfrentarán la tarea de dar conferencias en congresos internacionales con una visión paisajística y amplia de sus líneas de investigación o serán invitados como Keynote speakers a simposios y seminarios de prestigio, incluso fuera del campo de su especialidad. Eso sí, siempre dentro del ámbito de la comunidad científica, entendida como aquellas personas que -de una u otra forma- conocen de esto de hacer experimentos, validarlos con la indispensable estadística, publicarlos en revistas de impacto, aunque no sean exactamente científicos de su área de investigación.

¿Pero qué pasa cuando los científicos intentan salir de ese lugar en el que se manejan como peces en el agua y se trata de explicarle a "la gente", a posibles inversores que podrían financiar alguna de sus investigaciones aplicadas, o a los políticos de turno la importancia de su campo de estudio?

Del cuaderno de laboratorio al seminario interno, del seminario interno al abstract para el congreso, del abstract al paper, del paper al informe de avance, del informe de avance al pedido de financiamiento. Independientemente de en qué etapa de la carrera científica se encuentre un investigador, en todo este largo proceso de convertirse en científico, no llegaría a ninguna parte sin las adecuadas habilidades de comunicación. Más aún, nadie duda que para llegar a ser el orador principal en un evento científico internacional hace falta ser un buen comunicador, además de un buen científico. Entonces, ¿por qué la comunicación se nos presenta como algo completamente alejado del quehacer científico, salvo que estemos hablando de divulgadores de reconocida popularidad como Sagan, Cousteau, Paenza o Golombek ? Esto no siempre fue así. 
Hasta finales del siglo XIX, la separación entre ciencia y sociedad no era tan evidente como en la actualidad. Al contrario, existía entre ambas esferas una comunicación y apoyo mutuo que hacía difícil establecer una demarcación tan acentuada como la que existe hoy en día. De hecho, durante la revolución del siglo XVII, la corriente científica dominante, representada por la Royal Society, sostenía el carácter público de la ciencia. Se insistía en que para que una práctica fuera científica era necesaria la presencia del público. Más aún, muchas veces se asociaba la ausencia del público a la no cientificidad del experimento en cuestión.

De más está decir que no se puede afirmar que esta forma de práctica científica estuviese abierta a todos los miembros de la sociedad. El público presente en esas sesiones era cuidadosamente seleccionado e instruido. De todos modos, es interesante señalar que las primeras sociedades y academias científicas incluían no solo investigadores o practicantes, sino sobre todo personas interesadas en los resultados de las investigaciones.

Por ejemplo, si examinamos el catálogo de fellows de la Royal Society entre 1660 y 1700, encontraremos que algunas de las categorías utilizadas para describirlos eran: cortesano, ciudadano erudito de Londres, etc. Este carácter amateur del investigador científico también resulta evidente cuando se analiza la lista de veinticuatro filósofos naturales activos hacia 1770, sus intereses científicos y su ocupación o medio de ganarse la vida. Solo tres de los veinticuatro eran profesores de la materia objeto de su interés investigador.

En este contexto, no es de extrañar que en su célebre Diálogo sobre los dos grandes sistemas del mundo (1633), Galileo haya empleado la lengua vernácula para difundir una obra de contenido científico en la que defendía las tesis copernicanas prohibidas por la Iglesia católica en 1616. Con la clara finalidad de que pudieran entenderla los legos, Galileo se convierte en el primer gran divulgador científico de la historia.

Hoy quedó atrás la posibilidad de acceder a las pruebas y demostraciones que el público disfrutaba en otro tiempo, precisamente porque la ciencia se fue alejando de la esfera pública, sobre todo a lo largo del siglo XX.

En la actualidad, el 'aval científico' viene de la mano de instituciones, científicos, técnicas o procesos muy sofisticados que, muchas veces, solo pueden ser comprendidos dentro del contexto de la comunidad científica. Una comunidad que fue progresivamente erigiendo fronteras más precisas, dividiendo el campo entre especialistas (la ciencia) y legos (la sociedad).

Tan es así que, según algunos autores, este distanciamiento del público: «fue una de las condiciones necesarias para la producción de conocimiento propiamente científico. En contraste, allí donde la ciencia siguió influida sustancialmente por intereses públicos, el conocimiento objetivo y fiable se vio comprometido» (Shapin, 1990).

De alguna manera, podríamos decir que así como la ciencia se fue alejando de la sociedad a través de largo proceso histórico en donde dejó de ser un ámbito amateur para convertirse en un campo profesionalizado y que se desarrolla en el marco de instituciones cuya pertenencia requiere un largo entrenamiento, el aprendiz de científico debe hacer lo mismo si quiere pertenecer al pequeño círculo de privilegiados. 
Así como para "entrar" hay que andar un camino largo que es indispensable para convertirse en parte de la tribu, para saltar el llamado gap y comunicarse con el público es preciso desandar el camino, al menos en parte. Romper la estructura del paper, darle estructura narrativa al abstract, decodificar en clave humana el cuaderno de laboratorio. $Y$ sobre todo, darle un contexto de mundo real a todo aquello que ocurrió en la situación controlada del laboratorio. En este proceso de adaptación del discurso, resulta indispensable tener en cuenta que será preciso cambiar el enfoque hacia los posibles intereses, expectativas y contextos socioculturales de las audiencias. De alguna manera, será necesario ponerse nuevamente en contacto con aquel aprendiz de científico cuando dio sus primeros pasos en el camino de la ciencia.

\section{No todo es periodismo científico}

"¿Compiten los científicos-divulgadores con los periodistas y divulgadores profesionales?" se preguntaba el responsable de la Cátedra de Cultura Científica de la Universidad del País Vasco, Juan Ignacio Pérez en el VI Congreso de Comunicación Social de la Ciencia, desarrollado en la ciudad de Córdoba (España) en noviembre de 2017. Una pregunta que sintetiza un clásico debate que para ciertos sectores del periodismo científico parece no tener fin. La clave para responder a este interrogante bien podría resumirse en la respuesta que dio la periodista especializada en ciencia y salud argentina Nora Bär: "Lo que hacen unos y otros, no es lo mismo". En efecto, las actividades de popularización de la ciencia, denominadas por la jerga anglosajona como public engagement ${ }^{5}$, abarcan un amplio rango de actividades que incluyen desde dar charlas divulgativas en ámbitos escolares, dar entrevistas a periodistas científicos en periódicos, radio o televisión, escribir libros de divulgación, artículos de opinión en diarios o revistas de interés general, participar en debates públicos sobre temas científicos, actuar como voluntarios expertos en un panel de consenso o en un café científico, colaborar con organizaciones no gubernamentales (ONGs) u otro tipo de asociaciones como asesores o activistas, entre otros ejemplos.

Así, resulta evidente que si bien periodistas especializados y científicos divulgadores pueden estar compitiendo por los espacios, esto sólo ocurre cuando están ocupando posiciones en el ejercicio del periodismo, pero no en otros ámbitos de la comunicación pública de la ciencia, en los que se requiere un nivel de experticia elevado en cuanto al tema científico a divulgar.

De todos modos, si bien la comunicación es una tarea medular en la actividad científica, no todos los científicos son iguales a la hora de comunicar los resultados de sus investigaciones. Mucho menos, de cara al gran público.

Descontando el talento natural, que o se tiene o no se tiene, es probable que, como dicen por allí, "la práctica haga al maestro". Si aceptamos que existe una continuidad entre el cuaderno de laboratorio y el escenario de una charla TED, bien podría pensarse que un científico con más trayectoria ha desarrollado más habilidades de comunicación simplemente porque tiene más "tablas". Así, con un mínimo sentido de la observación acabaremos concluyendo algo que está a la vista de cualquiera: son esos científicos, los peces gordos, quienes con mayor frecuencia hacen de divulgadores. Pero, ¿por qué?

¿Será que un científico más avanzado en su carrera ya ha demostrado que tiene la capacidad de hacer buenos experimentos y que además sabe comunicarlos a sus pares y al gran público? ¿O quizás es porque alguien con una carrera sólida tiene que dar menos explicaciones a un jefe 
que un becario de iniciación y puede "darse el lujo" de realizar tareas de divulgación? ¿Acaso alguien en las instancias superiores de su carrera tenga cosas más interesantes para decir?

Puede que sí o puede que no. Lo cierto es que, según afirman la gran mayoría de los estudios en este campo, y muy contrariamente al mito de la saganización ${ }^{6}$ de los científicos que divulgan, a más alto estatus dentro de la organización y mayor productividad científica mayor es la probabilidad de interacción con los medios de comunicación y de participación en actividades de divulgación.

Según los especialistas, esta observación -que se constata en diversas latitudes- es probablemente el resultado de dos fenómenos. Por un lado, la preferencia de los periodistas por fuentes de alta reputación y la preferencia de los departamentos de prensa y relaciones públicas de las instituciones científicas de dar publicidad a los investigadores líderes de sus organizaciones. Por el otro, al privilegio formal o implícito de los jefes de unidades de investigación o de los investigadores principales de los proyectos, de representar a sus grupos de trabajo en los medios y frente al público en general.

Al parecer, no todas las actividades de divulgación tienen el mismo brillo. Por ejemplo, según revela un estudio realizado sobre investigadores del CONICET en Argentina, los científicos junior y los senior no realizan el mismo tipo de tareas de divulgación (Kreimer y cols., 2011). Mientras las actividades prestigiosas tales como las entrevistas en periódicos y televisión son más frecuentes entre los popes, las actividades vinculadas al trabajo con escolares o a jornadas de puertas abiertas son realizadas por investigadores en los primeros estadios de su carrera, algo que no necesariamente es decisión de los científicos.

Los autores del citado estudio, interpretan estas observaciones desde dos perspectivas. Por un lado, debido a la preferencia de los investigadores senior por formar parte de actividades de difusión en los medios de comunicación frente a la participación en actividades dirigidas a un público más "llano". Por el otro, sugieren que los periodistas usualmente requieren la opinión de expertos en ciertas áreas emergentes (cambio climático, organismos genéticamente modificados, epidemias, energía, etc.) donde los "expertos" suelen ser los más prestigiosos y los de más alta jerarquía.

Al margen de estos resultados, también habría que decir que las actividades dirigidas a ese público más "llano" compuesto por escolares y gente de a pie, generalmente tienen por objetivo acercar la ciencia que se hace día a día en el laboratorio. Entonces, ¿qué mejor que escuchar a quien efectivamente lleva la mayor carga experimental?

Bien sabemos que, así como se desarrollan más y mejor las habilidades de comunicación en la medida en que avanzan en la carrera científica, los científicos senior se van alejando en la misma medida de la mesada para ocupar su tiempo en otro tipo de tareas como el pedido de subsidios, la gestión de fondos, la formación de recursos humanos y las actividades de dirección. Sí, menos mesada y más escritorio.

Por otro lado, no es del todo cierto que siempre los "escolares" sean un público de segunda para los científicos que lideran los llamados cutting-edge projects. Si de apoyo se trata, en el 
Reino Unido existen iniciativas como el programa Researchers in Residence financiado por pesos pesados como la Wellcome trust y los UK Research Councils. Se trata de una iniciativa que propone acercar a las escuelas secundarias a investigadores líderes de proyectos innovadores. Durante su estancia, que se extiende entre 2 y 3 días, los investigadores trabajan en conjunto con los profesores a fin de ofrecer actividades que hagan la materia y la investigación relevantes e interesantes para los alumnos.

Asimismo, también en el Reino Unido, el programa Perspectives de la British Science Association busca implicar a jóvenes investigadores en actividades de comunicación en las que explican el contexto social de su trabajo en el laboratorio. ¿Por qué los más jóvenes? Para influir, desde una política pública, en cómo los científicos del futuro pensarán acerca de su investigación y cómo la comunicarán.

Pero, más allá del prestigio en juego, es posible que un investigador senior tenga efectivamente una visión más paisajística de su ciencia y sea más "apropiado" cuando se trata de dar visiones y opiniones globales para una entrevista. En cambio, un científico en las primeras etapas de su carrera seguramente tendrá la mesada más fresca y al día para contarle su cotidianeidad en el laboratorio a un grupo de escolares o a quien quiera escucharlo.

Sin duda, cada etapa tiene sus pros y sus contras de cara a las diversas actividades de divulgación. Pero, lo que definitivamente marca la diferencia es si el esfuerzo como científico que divulga es un esfuerzo individual, vocacional y no reconocido o si detrás de sus espaldas existe una institución o un programa que lo apoyan.

\section{Los científicos como narradores de historias}

Como decíamos al principio, la percepción de los aspectos negativos que plantea el desarrollo de la ciencia y la tecnología actual se ha vuelto - en las últimas décadas- más evidente en la sociedad. Esto resulta particularmente visible entre grupos sensibilizados como el movimiento ecologista, los grupos antiglobalización o los grupos pacifistas. Así, tanto la comunidad científica como los gobiernos comienzan a reconocer la necesidad de establecer un nuevo diálogo entre ciencia y sociedad. ¿Qué hacer con el desarrollo de la energía nuclear, los organismos genéticamente modificados o la investigación con células madre?

En un punto, parecería que cuanto más avanza la ciencia, la sociedad más se resiste. De hecho, para muchos científicos y responsables de política científica, la movilización del público contra algunas de estas cuestiones se basa en una resistencia a los avances de la ciencia y la innovación tecnológica. De acuerdo a esta interpretación, la principal causa de las posturas escépticas y hostiles hacia la ciencia sería un conocimiento insuficiente de los avances científicos por parte del público.

Sobre la base de este supuesto -conocido como 'modelo del déficit' de la comprensión pública de la ciencia, dado que hace hincapié en el déficit de conocimiento del público frente al de los especialistas- muchas instituciones han diseñado y promovido programas destinados a atenuar esta distancia a través de actividades de divulgación y comunicación de la ciencia al público general. 
Dentro de este esquema, la mente del no experto se concibe como un cubo vacío en el cual los hechos de la ciencia pueden y deberían ser vertidos al estilo de una aguja hipodérmica que inyecta conocimiento.

Pero este modelo, no solo asume que el conocimiento científico es fijo y certero, sino que, además, presupone un público en general indefinido y homogéneo. En contraste, sabemos que la comprensión de la ciencia también depende de forma crucial del entorno social, es decir de los grupos y sus pautas culturales, históricas e institucionales, en el cual el conocimiento se vuelve operativo (Irwin y Wynne, 1996). Y esto no es aprehensible desde la perspectiva del modelo de déficit cognitivo.

Esta crítica es el fundamento de nuevos modelos de comunicación científica que han surgido durante los últimos años. Basados en la idea de que la interpretación de hechos científicos no es un proceso inequívoco, los defensores de este punto de vista argumentan que el contexto social tiene un papel relevante en cuanto a la producción y al uso de los conocimientos científicos.

Desde esta perspectiva, los individuos no son entes vacíos y pasivos, sino que, por el contrario, procesan la información que reciben, negocian su significado y la reinterpretan e integran en el contexto de sus creencias, valores e intereses.

Así, los llamados modelos contextuales ven la generación de nuevos conocimientos públicos como un diálogo en el que, si bien los científicos tienen una serie de hechos acerca de la ciencia a su disposición, el público tiene un conocimiento local y una compresión personal de los problemas a resolver (Miller, 2001).

Tal como sugiere, la científica y divulgadora Susana Martínez-Conde en un reciente artículo (2017), "el mero hecho de proveer más información acerca de un tema determinado, no necesariamente cambiará las mentes o promoverá, por ejemplo, que una audiencia escéptica acepte la ciencia detrás del cambio climático". En este sentido, existe una línea emergente de trabajo dentro del campo de la comunicación pública de la ciencia que apunta a la utilización del storytelling y las narrativas a la hora de interactuar con públicos no expertos (Dahlstrom, 2014). Para algunos autores, aunque el uso de este tipo de aproximaciones a menudo suscita resistencia entre la comunidad científica, los formatos narrativos como estrategia de comunicación no deberían ser desestimados.

Acordamos con Bruner (2003) en que las narrativas pueden resultar "emblemáticas" por su capacidad de aportar conocimientos generales, además de sucesos singulares. De hecho, es esta capacidad la que las convierte en vehículos de conocimiento adecuados para diversas disciplinas, especialmente aquellas que lidian con complejidades históricas o que estudian fenómenos atravesados por una multiplicidad de factores causales, como aquellas vinculadas a la salud o el ambiente.

A lo largo de la historia, las narraciones sobre hechos reales o de ficción han sido utilizadas para compartir información, modificar creencias o inspirar comportamientos (Green y Brock, 2005). Tan es así, que incluso algunos autores afirman que el rasgo más distintivo del ser humano como especie, es su capacidad de contar historias (Morgan, 2010). La narración, enton- 
ces, aparece como una forma básica para que las personas conozcan y comprendan el mundo que las rodea.

Desde un punto de vista amplio, la comunicación científica podría ser comprendida como una labor de difusión cultural, equiparable con la de difusión de las bellas artes. Así, los objetivos a alcanzar desde esta perspectiva, se adscribirían más a la esfera de lo cultural que a la de lo pragmático o aplicado. De hecho, algunos comunicadores defienden el enfoque cultural de la divulgación de la ciencia por encima de otros enfoques.

Para el mexicano Bonfil Olivera (2003), por ejemplo, el objetivo de la comunicación de la ciencia es simplemente ponerla al alcance del público como una parte de la cultura, al igual que las artes, las tradiciones y los demás productos de la actividad humana, independientemente de su utilidad práctica. En sus propias palabras: «No se trata de enfocar la divulgación como una labor social, informativa, educativa, democrática ni periodística (mucho menos salvadora de vidas ni de patrias), sino interesante, placentera».

En cambio, otros comunicadores ponen el acento en generar productos de divulgación que complementen a la educación formal, como una ayuda para suplir las carencias de la enseñanza escolar. El principal objetivo desde este enfoque didáctico es la enseñanza de la ciencia. En esta línea, también podríamos clasificar a aquellas actividades de divulgación que pretenden lograr que más jóvenes estudien carreras vinculadas a la ciencia. En efecto, la falta de vocaciones científicas es un problema en el que coinciden tanto comunicadores como educadores.

También vinculada al mundo de los más jóvenes -aunque por qué no al de los adultos- encontramos las actividades de divulgación recreativas. Aquellas que buscan el puro placer de compartir la pasión de los científicos por la ciencia, sin otro propósito que deleitar al público. Algo así como un espectáculo popular o una actividad artística comúnmente clasificada como 'cultural'. Su objetivo es divertir y entretener, aunque al mismo tiempo fomenta el gusto por la ciencia.

En otra línea, podemos pensar en aquellas acciones de divulgación cuyo propósito es proveer al público de información oportuna acerca de los avances de las ciencias e interpretarlos, un tipo de divulgación que se enfoca en las noticias o en la novedad: el periodismo científico. Asimismo, muchos divulgadores tienen como fin combatir las creencias pseudocientíficas, las supercherías y supersticiones y difundir los hábitos de pensamiento crítico y objetivo propios de la ciencia.

Este tipo de divulgación que podríamos llamar escéptica, busca neutralizar ideas y creencias que ofrecen falsas versiones de la ciencia y fomentan el pensamiento mágico. Más aún, en el contexto de las nuevas relaciones entre ciencia y sociedad, resulta evidente que la finalidad democrática o social de la comunicación científica adquiere un papel preponderante.

En este caso, la divulgación científica puede concebirse como una labor social cuyo fin es democratizar el conocimiento, ponerlo al alcance de los ciudadanos al tiempo que los hace conscientes de la importancia de la ciencia y subraya su responsabilidad de participar en las decisiones relacionadas con la ciencia y la técnica que afecten a la sociedad. 
Pero, más allá de cuáles sean las finalidades de la comunicación científica, las estrategias narrativas constituyen herramientas potentes a la hora de explorar cómo llegar de manera clara y eficiente a las diversas audiencias, Como vimos a lo largo de este artículo, desde que dan sus primeros pasos en el laboratorio, los científicos están permanentemente contando historias: cómo hicieron sus experimentos, cómo arribaron a los resultados que están presentando, cuáles son sus hipótesis y cómo han llegado a ellas. Quizás el principal desafío de comunicar los resultados de sus investigaciones al gran público sea encontrar la manera de romper la estructura tradicional de comunicación con sus pares, fundamentalmente argumentativa, y convertirla en un formato narrativo.

\section{Notas}

1. El Manifiesto Russell-Einstein es un texto redactado por Bertrand Russell y apoyado por Albert Einstein, firmado en Londres el 9 de julio de 1955 que alertaba acerca de la peligrosidad de la proliferación del armamento nuclear, al tiempo que solicitaba a los líderes mundiales buscar soluciones pacíficas a los conflictos internacionales.

2. La primera de las Conferencias Pugwash tuvo lugar en julio de 1957 en la residencia particular del filántropo estadounidense Cyrus Eaton en el pueblo de Pugwash en Nueva Escocia, Canadá, de donde reciben su nombre genérico. Posteriormente se han ido celebrando en diferentes países.

3. El concepto Responsible Research and Innovation se refiere a los procesos de investigación e innovación que tienen en cuenta sus efectos y potenciales impactos en el ambiente y la sociedad. Este enfoque ha sido incluido en diversos programas marco de la Unión Europea y se ha desarrollado en publicaciones científicas y técnicas. Asimismo, existen diversas iniciativas que buscan aportar herramientas en este campo como el programa RRI Tools: https://www.rri-tools.eu/

4. La expresión anglosajona 'publish or perish' (publica o perece) se refiere a la presión creciente que sufren los científicos por ver publicados sus trabajos y poder, así, mantenerse en un sistema cada vez más competitivo por los espacios y los recursos.

5. La expresión "Public engagement" describe un conjunto de formas en el que la actividad y los beneficios de la educación superior y la investigación científica pueden ser compartidos con el público. La expresión "engagement" es por definición un proceso de dos vías que implica interacción y escucha recíproca, con el objetivo de generar un beneficio mutuo.

6. Durante la década de 1990 se difundió entre el mundo académico la idea de que como consecncia de dedicar más tiempo a la divulgación que a investigación, los científicos podían perder oportunidades. Así, por ejemplo, se difundió la idea de que Sagan perdió la oportunidad de ingresar como numerario en la universidad de Harvard y en la Academia Nacional de Ciencias de los Estados Unidos como consecuencia de su actividad divulgativa. Sin embargo, la producción científica de Sagan se había mantenido en los mismos niveles. Este tipo de situaciones, que son relativamente comunes entre científicos que se dedican además a la divulgación de la ciencia y se exponen a la opinión pública, se conocen como «efecto Sagan» a raíz del caso del astrofísico.

\section{Referencias}

- $\quad$ ALCÍBAR, M (2016). "¿Por qué la torre de marfil en ruinas?". Disponible en: http://www. investigacionyciencia.es/blogs/ciencia-y-sociedad/98/posts/por-qu-la-torre-de-marfilen-ruinas-14786

- $\quad$ BAUER, M; JENSEN, P (2011). The mobilization of scientists for public engagement. Public Understanding of Science. 20: 3.

- BONFIL OLIVERA, M. (2003): "Una estrategia de guerrilla para la divulgación: Difusión cultural de la ciencia". Ponencia para el 1er. Taller Latinoamericano Ciencia, comunicación 
y sociedad. Centro Nacional de Alta Tecnología, San José, Costa Rica, 24-26 de noviembre de 2003. Disponible en: http://www.cientec.or.cr/comunicacion/ponencias/MartinBonfil.pdf.

- $\quad$ BRUNER J. (2003) La fábrica de historias. Derecho, literatura, vida. Buenos Aires: Fondo de Cultura Económica.

- $\quad$ BRECHT, B. (2003): Vida de Galileo. Madre coraje y sus hijos. Alianza Editorial.

- $\quad$ BUSH, V. (1999): "Ciencia, la frontera sin fin. Un informe al Presidente, julio de 1945", Redes, n०14.

- $\quad$ CUTCLIFFE, SH. (2003): Ideas, máquinas y valores: los Estudios de Ciencia, Tecnología y Sociedad. Editorial Anthropos.

- $\quad$ DAHLSTROM MF (2014) Using narratives and storytelling to communicate science with nonexpert audiences. Proc Natl Acad Sci USA 111:13614-13620.

- $\quad$ DE SEMIR, V (2009). Master in scientific, medical and environmental communication. JCOM 8 (1), C02.

- $\quad$ EL MUNDO (28/11/2008): "Seminario en la Universidad Complutense. Internet es la gran oportunidad de la comunicación científica". Disponible en: http://www.elmundo.es/elmundo/2008/11/28/ciencia/1227898479.html

- EUROPEAN RESEARCH ADVISORY BOARD (EURAB). (Junio 2007): EURAB Report and Recommendations on "Research and Societal Engagement". Disponible en: http://ec.europa.eu/research/eurab/pdf/eurab 07013 june 202007 en.pdf

- GONZÁLEZ GARCÍA, M.; LÓPEZ CEREZO J.; LUJÁN LÓPEZ J. L. (1996): Ciencia, tecnología y sociedad: una introducción al estudio social de la ciencia y la tecnología. Madrid, Tecnos.

- GREEN, M. C. Y BROCK, T. C. (2005). Persuasiveness of narratives. En Brock, T. C. y Green, M. C. (Eds.). Persuasion. Psychological insights and perspectives (pp. 117-142). Thousand Oaks, CA: Sage (2a edición).

- HILGARTNER, S (1990). The Dominant View of Popularization: Conceptual Problems, Political Uses. Social Studies of Science, Vol. 20 (3): 519-539.

- $\quad$ IRWIN, A; WYNNE, B. (1996): Misunderstanding Science?: The Public Reconstruction of Science and Technology. Cambridge, Cambridge University Press.

- $\quad$ KREIMER, P; LEVIN, L; JENSEN, P (2011) Popularization by Argentine researchers: the activities and motivations of CONICET scientists. Public Understand. Sci. 20(1): 37-47

- LÓPEZ CEREZO, J. A. (1999): "Los estudios de ciencia, tecnología y sociedad". Revista Iberoamericana de Educación, n²0. En formato digital: http://www.rieoei.org/rie20a10.htm

- $\quad$ MARTÍNEZ-CONDE, S; MACKNIKA, SL (2017). Finding the plot in science storytelling in hopes of enhancing science communication. Proc Natl Acad Sci USA, vol. 114 (31): $8127-$ 8129

- MENDIZÁBAL, V.E. (2013). "6 ediciones, 200 alumnos, 30 ciudades" Panel: Especializaciones y posgrados en el área de Comunicación Pública de la Ciencia. $3^{\circ}$ Congreso Internacional de Comunicación Pública de la Ciencia: http://www.fcpolit.unr.edu.ar/programa-de-actividades-copuci-2013/

- MENDIZÁBAL, V.E. (2016). Científicos puertas afuera: cómo salir del laboratorio y comunicar los resultados de las investigaciones a la sociedad. Simposio PCST "Comunicación científica como profesión: Formación, responsabilidades y roles", con sede en San José, Costa Rica del 28 al 30 de setiembre del 2016.

- MERTON, R. (1973): The Sociology of Science: Theoretical and Empirical Investigations. Chicago, University of Chicago Press. 
- $\quad$ MILLER, S. (2001): "Public understanding of science at the crossroads". Public Understanding of Science, n० 10 (págs. 1-6).

- MORGAN, M. (2010). Cultivation analysis and media effects. En Nabi, R. L. y Oliver, M. B. (Eds.). The Sage handbook of media processes and effects (pp. 69-82). Thousand Oaks, CA: Sage.

- NELKIN, D. (1996): "An uneasy relationship: the tensions between medicine and the media" en The Lancet, vol. 347, no 9015: 1600-1603. Londres.

- PETERS, HP (2013). Gap between science and media revisited: Scientists as public communicators. PNAS. Vol. 110, Supplement 3: 14102-14109.

- PETERS, HP.; BROSSARD, D.; DE CHEVEIGNÉ, S.; DUNWOODY, S.; KALLFASS, M.; MILLER, S.; TSUCHIDA, S. (2008): "Interactions with the mass media". Science, vol. 321, no 5886: 204-205.

- PEW RESEARCH CENTER (2015). How scientists engage the public. Disponible en: http:// www.pewinternet.org/2015/02/15/how-scientists-engage-public/

- $\quad$ REED, R (2001).(Un-)Professional discourse? Journalists' and scientists' stories about science in the media. Journalism. Vol. 2: 3 (279-298).

- ROYAL SOCIETY (1985). The Public Understanding of Science. Disponible en: https:// royalsociety.org/ /media/Royal Society Content/policy/publications/1985/10700.pdf

- SHAPIN, S (1990). Science and the Public. In Companion to the History of Modern Science, ed. R. C. Olby, G. N. Cantor, J. R. R. Christie, and M. J. S. Hodge, 990-1007. London: Routledge.

- $\quad$ WARTOFSKY, M.W. (1976). Introducción a la filosofía de la ciencia, Madrid, Alianza Universidad, Cap.15.

\section{Links de interés}

- $\quad$ Agencia de la Universidad Nacional de La Matanza: http://www.ctys.com.ar/

- Agencia de la Universidad Nacional de San Martín: http://www.agenciatss.com.ar/

- Agencia de noticias científicas de la Fundación Instituto Leloir: http://www.agenciacyta. com.ar/

- Agencia de noticias de la Facultad de Ciencias Exactas y Naturales de la Universidad de Buenos Aires: http://nexciencia.exactas.uba.ar

- Alan Alda Center for Communicating Science: http://www.centerforcommunicatingscience.org/

- Carrera de Especialización en Comunicación Pública de la Ciencia y la Tecnología de la Universidad de Buenos Aires: http://comunicacion.exactas.uba.ar/carrera-especializacion/

- $\quad$ Center for Public Engagement with Science http://www.aaas.org/pes

- Centro Cultural de la Ciencia: http://ccciencia.gob.ar/

- Communicating Science Workshops (AAAS): http://www.aaas.org/pes/communicating-science-workshops

- Consejo Nacional de Investigaciones Científicas y Técnicas: http://www.conicet.gov.ar/

- Cursos y Seminarios de formación (Fundación Esteve): http://www.esteve.org/actividades/seminarios-de-formacion/

- Diplomatura en Comunicación Científica del IDEC-Universitat Pompeu Fabra en Buenos Aires: http://ccs.upf.edu/tag/postgrado-en-comunicacion-cientifica/

- El gato y la caja: https://elgatoylacaja.com.ar/ 
- Especialización en Comunicación Pública de la Ciencia y Periodismo Científico de la Universidad Nacional de Córdoba: http://www.cpc.unc.edu.ar/

- Especialización en Divulgación de la Ciencia, la Tecnología y la Innovación de la Universidad Nacional de Río Negro: http://unrn.edu.ar/blogs/divulgacion-cientifica/

- Ministerio de Ciencia, Tecnología e Innovación Productiva: http://www.mincyt.gob.ar/

- Nanotecnólogos por un día: http://www.nanoporundia.org/web/

- Poper Stand up Científico: https://www.facebook.com/poperstandup/

- Royal Society Public Engagement Committee https://royalsociety.org/about-us/committees/public-engagement-committee/

- Science with and for Society: https://ec.europa.eu/programmes/horizon2020/en/ h2020-section/science-and-society

- Tecnópolis: http://www.tecnopolis.mincyt.gob.ar/

- TecTV: http://www.tectv.gob.ar

\section{CURRICULUM VITAE: VICTORIA E. MENDIZÁBAL.}

Bióloga y Doctora en Farmacología por la Universidad de Buenos Aires. Master en Comunicación Científica, Médica y Ambiental por la Universidad Pompeu Fabra. Profesora Titular del Seminario de Comunicación y Salud en la Facultad de Ciencias de la Comunicación de la Universidad Nacional de Córdoba (UNC), profesora colaboradora de la Especialización en Comunicación Pública de la Ciencia y Periodismo Científico y Co-Directora del Posgrado en Prácticas de Comunicación en Salud, también en la UNC. Investigadora interesada en el análisis de medios y contenidos en el campo de la comunicación de la biomedicina y de la salud; y en el análisis y el diseño de estrategias de comunicación entre los científicos y sus públicos. 